Int. J. Dev. Biol. 48: 57-61 (2004)

Developmental Expression Pattern

\title{
Identification of a second Xenopus Twisted Gastrulation gene
}

\author{
MICHAEL OELGESCHLÄGER ${ }^{1,2}$, UYEN TRAN ${ }^{1}$, KRISTINA GRUBISIC² and EDWARD M. DE ROBERTIS*,1 \\ ${ }^{1}$ Howard Hughes Medical Institute, and Department of Biological Chemistry, University of California, Los Angeles, CA, USA \\ and ${ }^{2}$ Max-Planck Institute for Immunobiology, Freiburg, Germany
}

\begin{abstract}
Twisted Gastrulation (Tsg) is a secreted molecule which regulates BMP signalling in the extracellular space as part of an evolutionarily conserved network of interacting proteins. In Xenopus, maternal $x T s g$ mRNA can be found throughout the early embryo. After gastrulation, $x T s g$ is expressed as part of the BMP4 synexpression group until late tadpole stages. Here we report the identification of a second Xenopus Tsg gene (xTsg-2). Xenopus Tsg-2 is highly homologous to xTsg. In particular, amino acid residues which have been shown to be required for the binding of $x$ Tsg to BMP and to Chordin are conserved. The expression of Xenopus Tsg-2 mRNA was restricted to late stages of embryonic development; it was detected at tadpole stages in lateral plate mesoderm, neural crest, branchial arches and head mesenchyme. In microinjection experiments, the activity of $x T s g-2$ mRNA was similar to that of $x T s g$. We conclude that two Tsg genes act in distinct temporal and spatial territories in the course of Xenopus embryonic development.
\end{abstract}

KEY WORDS: twisted gastrulation, BMP, chordin, tolloid, Xenopus

The establishment of a BMP signalling gradient in the early embryo of vertebrates as well as invertebrates plays an important role in the determination of the dorsoventral (or back to belly) body axis. This gradient is generated in the extracellular space by an evolutionarily conserved system of interacting proteins involving the BMP antagonist Chordin that binds to BMP and prevents the binding of BMPs to their cognate receptors, metalloproteinases of the Xolloid/Tolloid family that cleave Chordin, and Twisted gastrulation (Tsg) a protein that binds both to BMP4 and Chordin (De Robertis et al., 2000). Several biochemical activities have been demonstrated for Tsg. First, Tsg facilitates the binding of Chordin to BMP leading to the formation of an inhibitory trimolecular complex (Oelgeschläger et al., 2000; Chang et al., 2001; Ross et al., 2001). Second, Tsg enhances the cleavage of Chordin by Xolloid/ Tolloid, generating proteolytic fragments of Chordin that can still bind to BMP and retain some anti-BMP activity (Larraín et al., 2001). Third, Tsg competes with the binding of the proteolytic cleavage products of Chordin to BMP (Oelgeschläger et al., 2000; Larraín et al., 2001). In loss-of-function situations XTsg can cooperate with Chordin (Ross et al., 2000; Blitz et al., 2003) and with BMP4 (Zakin and De Robertis, 2003).

Multiple Tsg genes have been identified in fruit flies. In Drosophila, Tsg is required for the formation of a narrow stripe of peak BMP activity in the dorsal midline of the early fly embryo (Ross et al., 2001; Eldar et al., 2002).

A second, highly homologous Tsggene has been reported to be inactivated in the crossveinless (cV) mutant that lacks crossveins in the adult wing (Vilmos, 2001). The formation of crossveins is preceded by a localized peak of BMP signalling that can be visualized by phospho-MAD specific antibodies (Conley et al., 2000). A third Tsg-like gene has recently been identified in the Drosophila genome. $d T s g 3$ encodes a protein consisting exclusively of the carboxy-terminal cysteine-rich domain and maps close to the shrew locus (Vilmos et al., 2001). Shrew is the last of the Drosophiladorsal-ventral zygotic mutations that remains to be identified (De Robertis and Sasai, 1996). Interestingly, we have demonstrated that the ventralizing (pro-BMP) activity of Tsg resides in the domain encoded by dTsg3 (Oelgeschläger et al., 2003b). shrew mutant flies, like tsgmutants, are unable to form the amnioserosa, the dorsal-most tissue of the early fly embryo that requires maximum BMP signalling (Ferguson and Anderson, 1992; Arora et al., 1992).

In zebrafish, a second Tsg gene expressed at later stages of development has been reported (Ross et al., 2001). However, in Xenopus, mouse, and human only one tsg gene has been identified to date (Graf et al., 2001; Nosaka et al., 2003). In mouse, Tsg is required for the formation of bone and thymus, and has been implicated in the regulation of T-cell differentiation (Graf et al., 2002; Nosaka et al., 2003). Tsg ${ }^{-1-}$ mice are viable and fertile, but are of small size and have kinky tails, osteoporosis, and other defects (Nosaka et al., 2003; Zakin and De Robertis, 2003).

Abbreviations used in this paper: BMP, bone morphogenetic protein; Tsg, twisted gastrulation gene; xTsg, Xenopus twisted gastrulation gene.

\footnotetext{
*Address correspondence to: Dr. Edward M. de Robertis. Department of Biological Chemistry, University of California, Los Angeles, CA 90095-1662, USA. Fax: +1-310-206-2008. e-mail: derobert@hhmi.ucla.edu
} 
B

A

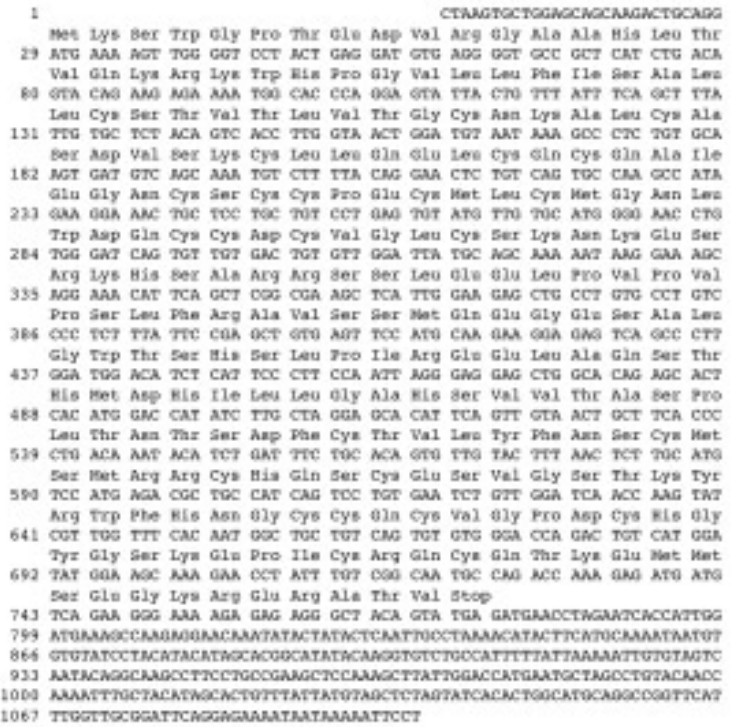

C

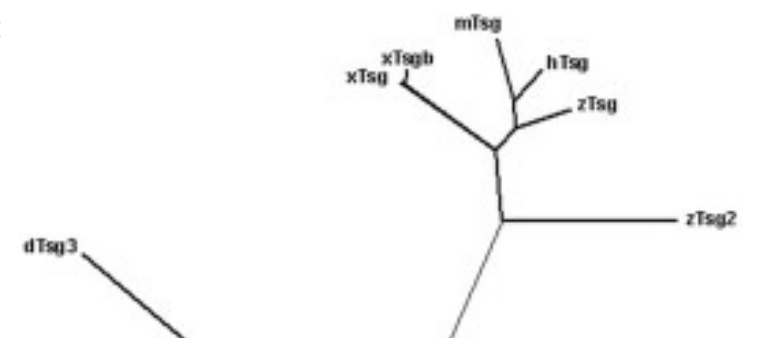

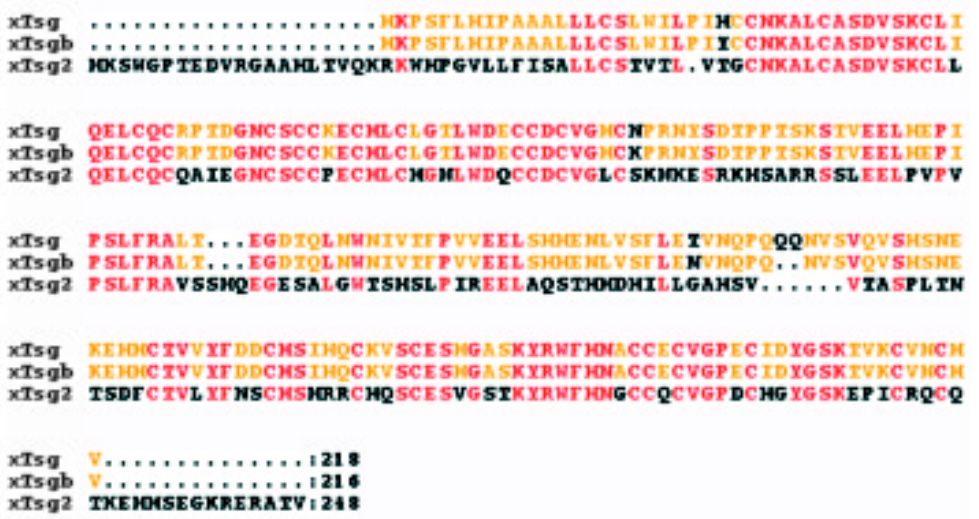

D

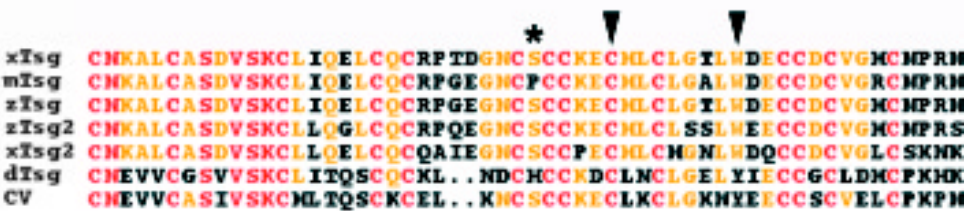

XTsg CTVVYT DCMS IHECKV SCE SIG A SKXRWFHACCE CVGPC IDYG SKTVKCVNC

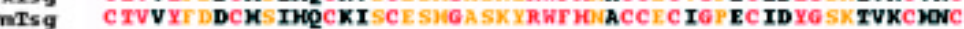

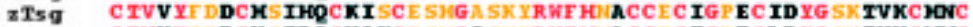

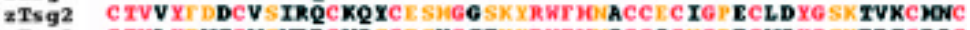

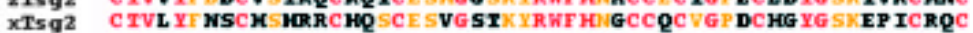
dTsg CTY IYYNSCIRANKCROOCE SUGASS YRWFHDGCC CVGEWCLWYG IOE SRCRGC dTsg CV

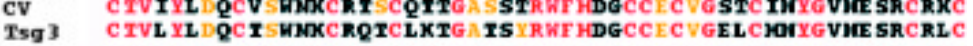

Fig. 1. Analysis of the xTsg2 cDNA and protein sequence. (A) Nucleotide and amino acid sequence of $x$ Tsg-2. (B) Alignment of the two pseudoalleles of xTsg with xTsg-2. (C) Dendrogram showing the phylogenic relations between the Xenopus (xTsg, xTsgb, xTsg-2), mouse (mTsg), human (hTsg), zebrafish (zTsg, zTsg-2) and Drosophila Tsg proteins (dTsg, cv/dTsg2, dTsg3). (D) ClustalWalignment of the indicated protein sequences demonstrating the conservation of amino acids required for ventralizing activity or chordin binding (arrowheads) and the position of an in vivo glycosylation site (asterisk). The accession numbers of the sequences used for the alignments were for xTsg, AF245221; xTsgb, AF279246; xTsg-2, BJ075535; mTsg, AAG00605; hTsg, NP_065699; zTsg, AAK27324; zTsg2, AAK13255; dTsg, A53836; cv, CG12410; dTsg3, CG11582.

Interestingly, when one copy of BMP4 is removed as well, Tsg/-;BMP4+/- embryos develop holoprocencephaly (Zakin and De Robertis, 2003). Since BMP4 becomes haploinsufficient in the absence of Tsg this shows that Tsg promotes BMP4 activity during forebrain development in the mouse. However, the overall phenotype of the mouse Tsg mutation was surprisingly mild. In this paper we report the isolation of a second Tsg gene in Xenopus.

\section{Results and Discussion}

A new Xenopus Tsg-like gene was identified in the public EST database (Accession \# BJ075535; Fig. 1A) and sequenced on both strands. It encodes a $27 \mathrm{kD}$ secreted protein with a potential signal peptide cleavage site at position $44\left(V_{T G}^{44}-C^{45} N\right)$ (Fig. 1B).
Xenopus laevisis an allotetraploid species in which two pseudoalleles can be found for many genes (Kobel and Du Pasquier, 1986). However, the new gene, designated $x T s g-2$, does not appear to encode a pseudoallele of Tsg. Xenopus pseudoalleles share strong sequence identity at the nucleic acid level with over $90 \%$ amino acid identity in the coding region, although in the untranslated regions of the mRNA the sequence can be more diverse (Oelgeschläger et al., 2003b). For Xenopus, two XTsgpseudoalleles have already been reported (Oelgeschläger et al., 2000; Scott et al., 2001; Blitz et al., 2003). They share $90 \%$ amino acid identity including the signal peptides (Fig. 1B). The $x$ Tsg-2 gene shares only $50 \%$ amino acid identity with the two $T s g$ pseudoalleles, even after excluding the signal peptide (Fig. 1B). The similarities are mainly found in the $\mathrm{N}$ - and $\mathrm{C}$-terminal cysteine-rich domains that 


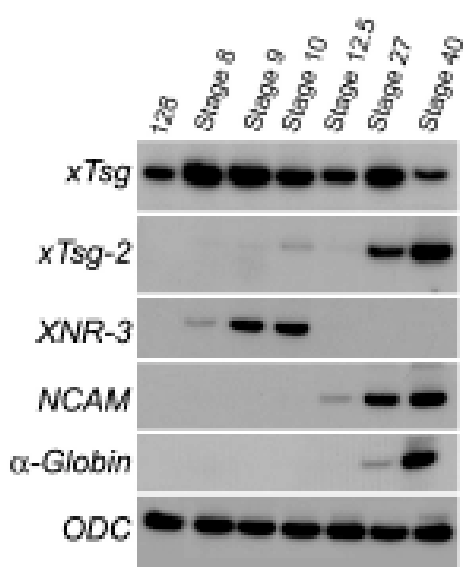

Fig. 2. Time course of $x T s g$ and $x T s g-2$ gene expression. mRNA samples were analyzed by RT-PCR at the indicated developmental stages. Xenopus Tsg mRNA can be detected throughout development, whereas $x$ Tsg- 2 expression starts at tailbud stages. Other markers shown are XNR-3, which responds specifically to the early $\beta$-catenin signal in Xenopus; NCAM, which marks neural tissue; $\alpha$-globin, which marks ventral mesoderm and blood differentiation; ODC lornithine decarboxilase) serves as an mRNA loading control.

are required for the binding of $\mathrm{xTsg}$ to $\mathrm{BMP}$ and to Chordin (Oelgeschläger et al., 2000; Oelgeschläger et al., 2003a). We compared the $\mathrm{xTsg}-2$ sequence to the Tsg proteins identified in other species and found that it is a distant member of the vertebrate Tsg family (Fig. 1C).

We recently identified amino acid residues required for the binding of Tsg to BMP and to Chordin (Oelgeschläger etal., 2003a). Mutations in the $\mathrm{N}$-terminal domain of Tsg were found to enhance its ventralizing (BMP-promoting) activity and to interfere with BMP binding. As seen in Fig. 1D, these amino acids (indicated by arrowheads) are conserved in XTsg-2 as well as in Tsg proteins isolated from various vertebrate and invertebrate species. In the C-terminus, site-directed mutagenesis of C180 and C197 of XTsg into Alanine had the opposite effect, loss of the ventralizing activity, which correlated with a loss of the ability of $x T s g$ to bind to Chordin (Oelgeschläger et al., 2003a). These residues are conserved in all Tsg proteins listed in Fig. 1D (arrowheads). We also identified an in vivo glycosylation site (S54) in xTsg that is conserved in XTsg-2 and most of the other Tsg proteins, with the exception of Drosophila and mouse Tsg (Fig. 1D, asterisk).

We next compared the expression patterns of $x T s g$ and $x$ Tsg-2. Xenopus Tsg mRNA was detected in RTPCR analysis from the onset of embryonic development until late tadpole stages while $\times$ Tsg-2mRNA could not be detected until early tadpole stages (Fig. 2). In agreement with the RT-PCR analyses, $x$ Tsg-2 transcripts became detectable by in situhybridization at early tailbud tadpole stages, when transcripts could be detected in head mesenchyme, branchial arches and lateral plate mesoderm (Fig. 3 A,C). At later stages the signal increased significantly and expression became clearly visible in the head region, lateral plate mesoderm and neural crest (Fig. 3B). When embryos were cleared in Murray's solution, higher staining in the midbrain-hindbrain isthmus became apparent (Fig. 3D). In the branchial arches, $x$ Tsg-2was expressed in the neural crest derived central core, but not in the pharyngeal pouches and ectoderm (Fig. 4 A,B). The neural tissue showed weak signals in the dorsal margin of the retina, the ventricular (proliferative) zone of most of the hindbrain, and in the midbrainhindbrain border (Fig. 4C). Expression in head mesen- chyme extended throughout head structures, surrounding the eye (Fig. 4C) and otic vesicle, but excluding the cement gland (Fig. 4B). At the level of the trunk, $x$ Tsg-2 was expressed in the lateral plate mesoderm (Fig. 4F), at weaker levels in the pronephros (Fig. 4D) and pronephric duct (data not shown). In addition, staining in neural crest cells that migrate into the dorsal fin was visible (Fig. 4F).

When compared to $x T s g-2, x T s g$ was found to have a very different expression pattern. $x T s g$ is expressed maternally and ventrally during gastrulation (Oelgeschläger et al., 2000). At early tadpole stages, strong expression of $x T s g$ was found in the dorsal eye, the heart anlage and the peri-anal region (Oelgeschläger et al., 2000) (Fig. 3E). At later stages, the posterior expression domain becomes restricted to the posterior wall (ventral mesoderm, Gont et al., 1993) of the tip of the tailbud (Fig. 3F). We conclude that $x T s g-2$ and $x T s g$ are genes with distinct expression patterns. $x$ Tsg-2is expressed at tadpole stage in the mesenchyme of the head, branchial arches and lateral plate mesoderm. Expression of $x$ Tsgis more extensive in earlier development (Oelgeschläger et al., 2000), while $x T s g-2$ is expressed at tadpole stages.

The high homology between $x$ Tsg and $x T s g-2$, especially the conservation of amino acids involved in the interaction of Tsg with BMP and Chordin, implied that $x T s g-2$ and $x T s g$ might have similar biological activities. We tested $x T s g-2$ activity by microinjecting synthetic mRNA and found that the phenotype observed was undistinguishable to that of $x T s g$ (Fig. 5). Microinjection of
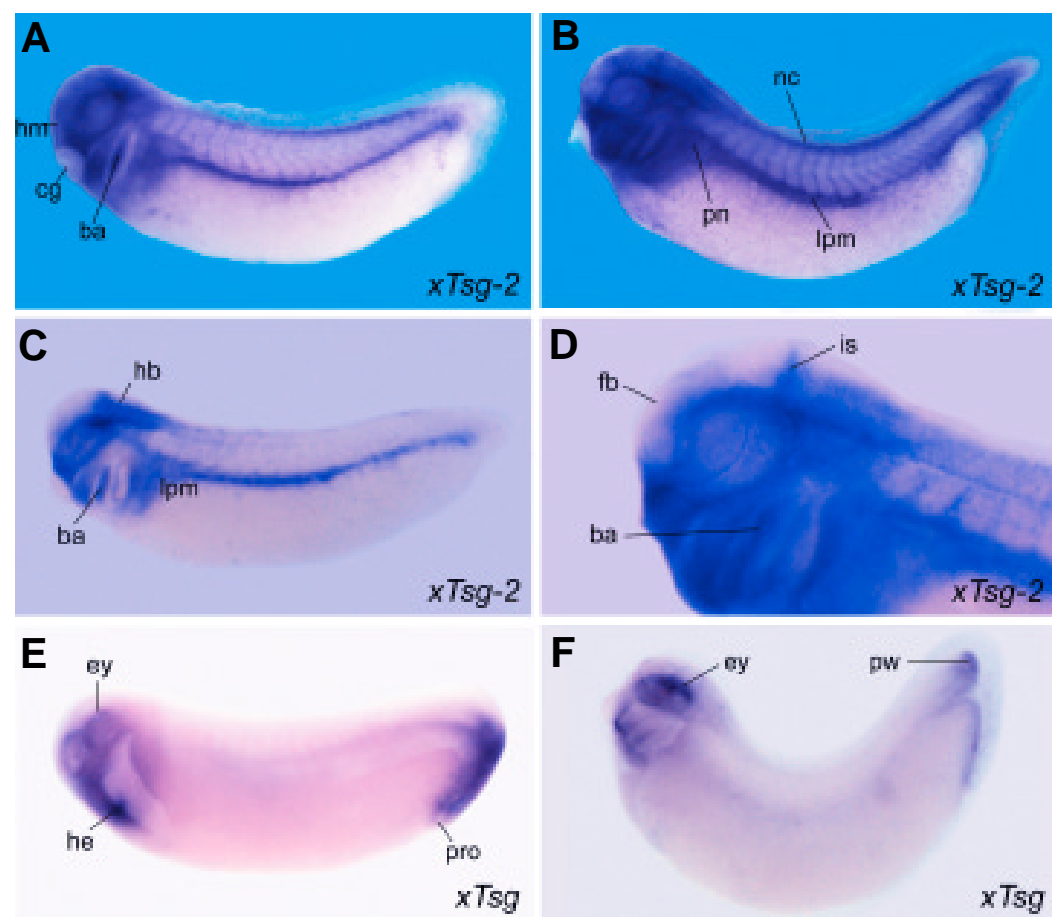

Fig. 3. Whole-mount in situ hybridization analysis of $x T s g-2$ and $x T s g$ expression. (A,C) xTsg-2 expression at stage 27 and (B,D) stage 33 embryos showing expression in the branchial arches (ba), neural crest (nc), lateral plate mesoderm (lpm) and head mesenchyme (hm). Embryos in C and D were made transparent in Murray's Clearing Solution (2:1 benzyl benzoate: benzyl alcohol) to visualize the expression of $\mathrm{xTsg}-2$ localized to the midbrain-hindbrain border, the isthmus (is). D is a close up of the embryo in B. (E) Stage 27 and (F) stage 33 embryos stained for XTsg and cleared in Murray's solution reveal expression in the dorsal eye (ey), heart anlage (he) and proctodeum (pro), as well as in the posterior wall (pw) of the tailbud. cg, cement gland; fb, forebrain; $h b$, hindbrain; pn, pronephros. 

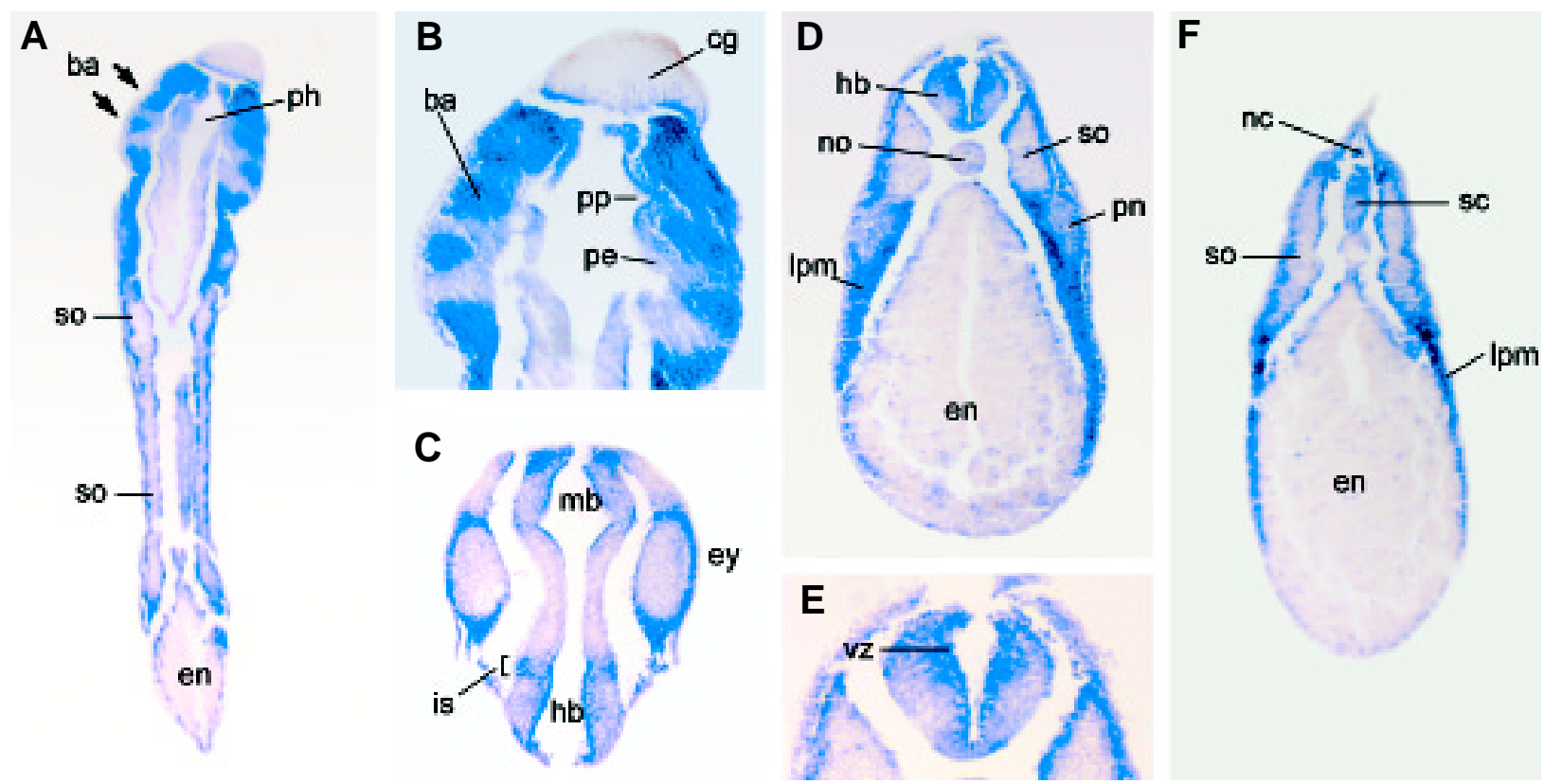

Fig. 4. $x$ Tsg-2 is expressed in the mesenchyme of the branchial arches, lateral plate and other tissues. Histological sections of whole-mount in situ hybridization. (A) Coronal section of an embryo at stage 33 showing expression of xTsg-2 in the branchial arches (ba), but not in the pharynx (ph). (B) Close up of $(A)$ indicating the expression of XTsg-2 is restricted to the branchial arches (ba), but devoid of detectable expression in the pharyngeal pouches (pp) consisting of pharyngeal endoderm (pe) as well as the cement gland (cg). (C) XTsg-2 expression is observed at low amounts in the eye (ey), but strong in the surrounding head mesenchyme. The isthmus (is) is stained in this coronal section. (D) Transverse section of a stage 33 embryo at the level of the trunk showing xTsg-2 expression in the lateral plate mesoderm (lpm) and hindbrain (hb) and low levels in the pronephros (pn). The somites (so), endoderm (en) and notochord (no) are not stained. (E) Close up showing xTsg-2 expression in the ventricular (proliferative) zone (vz) of the hindbrain. (F) Transverse section showing xTsg-2 expression in the dorsal fin neural crest cells and dorsal spinal cord (sc).

$x$ Tsg-2mRNA induced the formation of an enlarged proctodeum that often detached from the trunk (Fig. 5C). This phenotype is seen in $x$ Tsg mRNA injections (Fig. 5B; Chang et al., 2001; Oelgeschläger et al., 2003a) and is also observed in embryos in which BMP signalling is modulated by a variety of agents (Beck et al., 2001; Oelgeschläger et al., 2003a). The results indicate that $x T s g-2$ and $x$ Tsg have similar overexpression phenotypes in Xenopus embryos.

The existence of a second Tsg gene with redundant functions could help to provide an explanation for why the loss-of-function
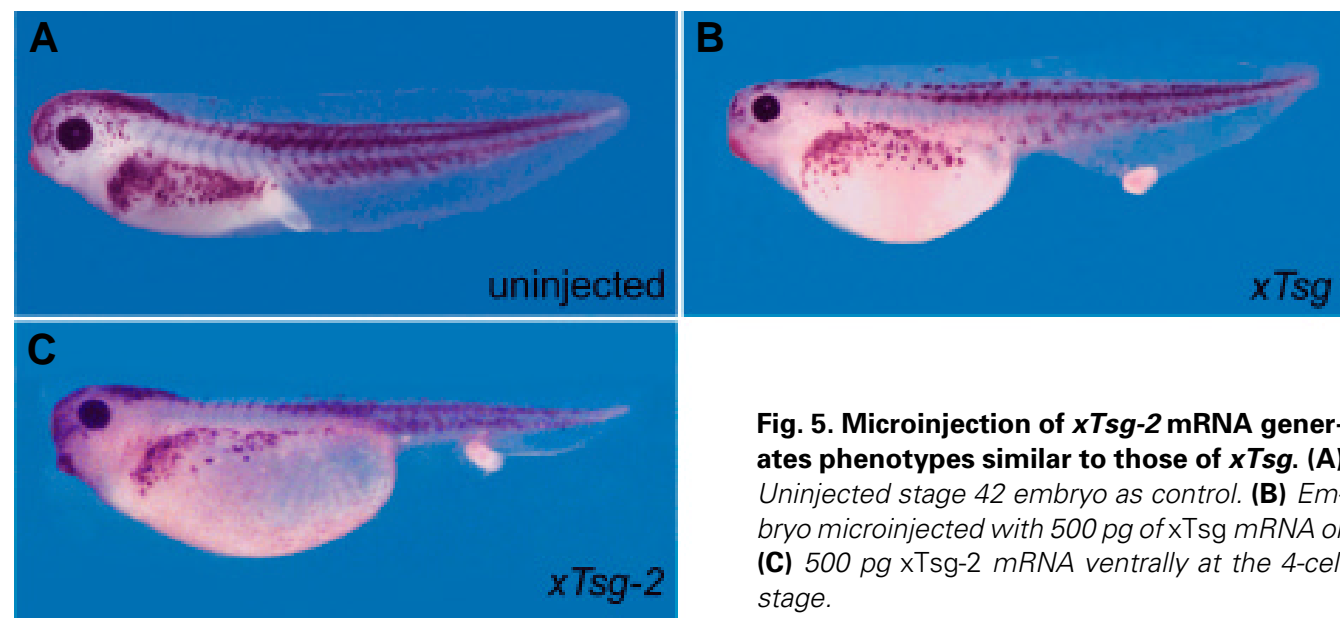

Fig. 5. Microinjection of $x T s g-2$ mRNA generates phenotypes similar to those of $x T s g$. (A) Uninjected stage 42 embryo as control. (B) Embryo microinjected with $500 \mathrm{pg}$ of XTsg mRNA or (C) $500 \mathrm{pg} \times \mathrm{Tsg}-2$ mRNA ventrally at the 4-cell stage. of mouse Tsg has only weak phenotypes (Nosaka et al., 2003; Zakin and De Robertis, 2003). We note that although the mouse and human genomes have been completely sequenced, some genes, particularly those consisting of small exons, are sometimes difficult to identify.

The expression of $x T s g-2$ did not overlap at any time with the expression of chordin, whereas the broad early expression of $x T s g$ and its specific expression later in the posterior wall of the tailbud may imply a functional interaction of xTsg with Chordin. Chordin is expressed in the posterior notochord or chordoneural hinge in the tailbud at early tadpole stages (Sasai et al., 1994). The expression domain of $x T s g-2$ in lateral plate mesoderm and neural crest adjoins the expression of Xolloid in the inner layer of the epidermis, and in the head region $x T s g-2$ overlaps with the expression of XBMP1(Goodman etal., 1998). A number of proteins that contain Chordin-like BMP-binding domains have been identified in vertebrates and for many of them a functional or biochemical interaction with TGF- $\beta$ or BMP has been demonstrated (García Abreu et al., 2002). Thus, it seems possible that $x T s g-2$ may act together with 
BMP and members of the Tolloid family of metalloproteases on other Chordin-related proteins at later stages of development. In future it will be important to identify the extracellular proteins that interact with $\mathrm{xTsg}-2$ in the course of embryonic development.

\section{Experimental Procedures}

\section{DNA constructs}

The constructs used for $x$ Tsg microinjections were previously described (Oelgeschläger et al., 2000). For $x T s g-2$, the coding region was amplified by PCR and cloned into the pCS2+ vector. For mRNA synthesis the pCS2-xTsg2 construct was linearized with Not and transcribed with SP6 RNA Polymerase using the mMessage mMachine Kit (Ambion). The sequence of the $x$ Tsg-2 cDNA described here has been deposited in GenBank under accession number BK001651).

\section{In situ hybridization and RT-PCR}

Xenopus embryos obtained by in vitro fertilization were maintained in Barth Solution (Sive et al., 2000) and staged according to Nieuwkoop and Faber (1994). Whole-mount in situhybridization for $x$ Tsg were performed as described (Oelgeschläger et al., 2000). For $x T s g-2$ antisense probe, a full length EST (Accession number: BJ075535) was linearized with EcoR, transcribed with T7 RNA polymerase and prepared as described (Oelgeschläger et al., 2000; and http://www.hhmi.ucla.edu/derobertis/ index.html). Embryos were made transparent in Murray's Clearing Solution (2:1 benzyl benzoate: benzyl alcohol). Histological sections of $x T s g$-2stained embryos were embedded in paraplast, sectioned at $25 \mu \mathrm{m}$, dewaxed, dehydrated and embedded in Permount. RT-PCR conditions and primer sets were previously described (Oelgeschläger et al., 2000; and http:// www.hhmi.ucla.edu/derobertis/index.html). For $x T s g-2$, the primer sets were 5'-ATG AAA TGG GGT CCT AC-3' (forward) and 5'-AGT TTC CTT CTA TGG CTT GG-3' (reverse).

\section{Acknowledgements}

We thank Drs. O. Wessely and J. Kim for comments on the manuscript. This work was supported by the National Institutes of Health (R37 HD2150216 to E.M.D.R) and the Max-Planck Society. E.M.D.R. is an Investigator of the Howard Hughes Medical Institute.

\section{References}

ARORA, K. and NUSSLEIN-VOLHARD, C. (1992). Altered mitotic domains reveal fate map changes in Drosophila embryos mutant for zygotic dorsoventral patterning genes. Development 114:1003-24.

BECK, C.W., WHITMAN, M. and SLACK, J.M. (2001). The role of BMP signaling in outgrowth and patterning of the Xenopus tail bud. Dev. Biol. 238: 303-314.

BLITZ, I.L., CHO, K.W. and CHANG, C. (2003). Twisted gastrulation loss-of-function analyses support its role as a BMP inhibitor during early Xenopus embryogenesis. Development 130: 4975-4988.

CHANG, C., HOLTZMAN, D.A., CHAU, S., CHICKERING, T., WOOLF, E.A., HOLMGREN, L.M., BODOROVA, J., GEARING, D.P., HOLMES, W.E. and BRIVANLOU, A.H. (2001). Twisted gastrulation can function as a BMP antagonist. Nature 410: 483-487.

CONLEY, C.A., SILBURN, R., SINGER, M.A., RALSTON, A., ROHWER-NUTTER, D., OLSON, D.J., GELBART, W. and BLAIR, S.S. (2000). Crossveinless 2 contains cysteine-rich domains and is required for high levels of BMP-like activity during the formation of the cross veins in Drosophila. Development 127: 3947-3959.

DE ROBERTIS, E.M. and SASAI, Y. (1996). A common plan for dorsoventral patterning in Bilateria. Nature 380: 37-40.

DE ROBERTIS, E.M., LARRAíN, J., OELGESCHLÄGER, M. and WESSELY, O. (2000) The establishment of Spemann's organizer and patterning of the vertebrate embryo. Nat Rev Genet. 1: 171-81.

ELDAR, A., DORFMAN, R., WEISS, D., ASHE, H., SHILO, B.Z. and BARKAI, N. (2002) Robustness of the BMP morphogen gradient in Drosophila embryonic patterning. Nature 419: 304-308.
FERGUSON, E.L. and ANDERSON, K.V. (1992). Localized enhancement and repression of the activity of the TGF- $\beta$ family member, decapentaplegic, is necessary for dorsal-ventral pattern formation in the Drosophila embryo. Development 114: 583597.

GARCÍA ABREU, J., COFFINIER, C., LARRAÍN, J., OELGESCHLÄGER, M. and DE ROBERTIS, E.M. (2002). Chordin-like CR domains and the regulation of evolutionarily conserved extracellular signaling systems. Gene 287: 39-47.

GONT, L.K., STEINBEISSER, H., BLUMBERG, B. and DE ROBERTIS, E.M. (1993). Tail formation as a continuation of gastrulation: the multiple cell populations of the Xenopus tailbud derive from the late blastopore lip. Development 119: 991-1004.

GOODMAN, S.A., ALBANO, R., WARDLE, F.C., MATTHEWS, G., TANNAHILL, D. and DALE, L. (1998). BMP1-related metalloproteinases promote the development of ventral mesoderm in early Xenopus embryos. Dev. Biol. 195, 144-157.

GRAF, D., TIMMONS, P.M., HITCHINS, M., EPISKOPOU, V., MOORE, G. ITO, T., FUJIYAMA, A., FISHER, A.G. and MERKENSCHLAGER, M. (2001). Evolutionary conservation, developmental expression, and genomic mapping of mammalian Twisted gastrulation. Mamm. Genome 124: 554-560.

GRAF, D., S. NETHISINGHE, D. B. PALMER, A. G. FISHER andMERKENSCHLAGER $M$. (2002). The developmentally regulated expression of twisted gastrulation reveals a role for bone morphogenetic proteins in the control of T cell development. J. Exp. Med. 196: 163-171.

KOBEL, H.R., and DU PASQUIER, L. (1986). Genetics of polyploidy Xenopus. Trends Genet. 2: 310-315

LARRAÍN, J., OELGESCHLÄGER, M., KETPURA, N.I., REVERSADE, B., ZAKIN, L. and DE ROBERTIS, E.M. (2001). Proteolytic cleavage of Chordin as a switch for the dual activities of Twisted gastrulation in BMP signaling. Development 128: 44394447.

NIEUWKOOP, P. D., and FABER, J. (1994). "Normal table of Xenopus laevis." Garland Publishing, Inc., New York.

NOSAKA, T., MORITA, S., KITAMURA, H., NAKAJIMA, H., SHIBATA, F., MORIKAWA Y., KATAOKA, Y., EBIHARA, Y., KAWASHIMA, T., ITOH, T. et al. (2003). Mammalian twisted gastrulation is essential for skeleton-lymphogenesis. Mol. Cell Biol. 23: 2969-2980.

OELGESCHLÄGER, M., LARRAíN, J., GEISSERT, D. and DE ROBERTIS, E. (2000). The evolutionarily conserved BMP-binding protein Twisted gastrulation promotes BMP signalling. Nature 405: 757-63.

OELGESCHLÄGER, M., REVERSADE, B., LARRAíN, J., LITTLE, S., MULLINS, M.C and DE ROBERTIS, E.M. (2003a). The pro-BMP activity of Twisted gastrulation is independent of BMP binding. Development 130: 4047-4056.

OELGESCHLÄGER, M., KURODA, H., REVERSADE, B. and DE ROBERTIS, E.M. (2003b). Chordin is required for the Spemann organizer transplantation phenomenon in Xenopus embryos. Developmental Cel/4: 219-30.

ROSS, J.J., SHIMMI, O., VILMOS, P., PETRYK, A., KIM, H., GUADENZ, K., HERMANSON, S., EKKER, S.C., O'CONNOR, M.B. and MARSH, J.L. (2001). Twisted gastrulation is a conserved extracellular antagonist. Nature 410: 423-424.

SASAI, Y., LU, B., STEINBEISSER, H., GEISSERT, D., GONT, L.K., and DE ROBERTIS E.M. (1994). Xenopus chordir. a novel dorsalizing factor activated by organizerspecific homeobox genes. Cel/79: 779-790.

SCOTT, I.C., BLITZ, I.L., PAPPANO, W.N., MAAS, S.A., CHO, K.W.Y. and GREENSPAN, D.S. (2001). Homologues of Twisted gastrulation are extracellular cofactors in antagonism of BMP signalling. Nature 410: 475-478.

SIVE, H. L., GRAINGER, R. M., and HARLAND, R. M. (2000). Early development of Xenopus laevis. A laboratory manual. Cold Spring Harbor Laboratory Press, Cold Spring Harbor, New York.

VILMOS, P., GAUDENZ, K., HEGEDUS, Z. and MARSH, J.L. (2001). The Twisted gastrulation family of proteins, together with the IGFBP and CCN families, comprise the TIC superfamily of cysteine rich secreted factors. Mol. Pathol. 54: 317-23.

ZAKIN, L. and DE ROBERTIS, E.M. (2003). Inactivation of mouse Twisted gastrulation reveals its role in promoting BMP4 activity during forebrain development. Development. 131: 413-424. 